- People with TB can continue treatment and normal activities when they are not infectious.

- If people with TB do not take their medication, they can become seriously ill, and may even die.

\section{ARETB PATIENTS ALWAYS INFECTIOUS?}

- People with TB of the lungs or throat can be infectious to others.

- People with TB in other parts of the body are not infectious.

- In most cases, after two weeks of taking medication, patients with TB disease will no longer spread germs.

\section{WHO DOTB PATIENTS HAVETOTELL ABOUT THEIR TB?}

Like any other illness, only the doctors and chest clinic staff need to know a person has TB.
The chest clinic staff will decide which 'contacts' need testing. 'Contacts' are often other family members, but sometimes may be close friends or work mates. Contact tracing is always done sensitively and confidentially.

\section{CHEST CLINIC SERVICES INCLUDE:}

- all the necessary contact tracing and screening

- the care and management of patients with TB.

Note:

- a referral from a doctor is NOT needed to attend a chest clinic.

- a Medicare card is NOT needed.

For more information, please contact your local Chest Clinic, Public Health Unit or Doctor.

\title{
COMMUNICABLE DISEASES, NSW: AUGUST 2000
}

\section{TRENDS}

This month we introduce four small changes in our presentation of notifications of communicable diseases:

- the title of this presentation will change from Infectious Diseases to Communicable Diseases;

- in Figure 6, a graph showing the number of cases of influenza diagnosed by virological techniques from six major public laboratories replaces our usual graph showing the number of cases of rubella. (Rubella notifications will continue to be reported in Table 1.) Voluntary reports of influenza are received from May through to September each year. The number of participating laboratories increased from four to six in 1998;

- to compensate for reporting delays, Figure 6 now includes a line in each graph showing predicted notifications after adjusting for likely reporting delays;
- Table 1 now includes a column for cases residing in prison (that is, within the Corrections Health Service).

The data in Table 1 and Figure 6 show:

- a decline in notifications of arbovirus infections (expected with the fewer mosquitoes as a result of colder weather);

- a rise in notifications of meningococcal disease (cases usually increase in mid-winter) serving as a reminder for clinicians to have an increased level of alertness for cases, to treat suspected cases early with intravenous antibiotics, and to notify suspected cases to the local public health unit;

- a rise in pertussis notifications in some areas of the State, notably New England, and a continuing high level in the Hunter;

- Influenza activity remains at low levels, with 17 influenza type A and 10 influenza type B diagnoses 
reported from viral laboratories in June.

\section{SHIGELLOSIS OUTBREAK AMONG INNER- SYDNEY MEN}

NSW Health and South Eastern Sydney Area Health Service are currently investigating an outbreak of shigellosis among inner-Sydney men who have sex with men. Shigellosis is a highly infectious bacterial infection affecting the bowel and is characterised by diarrhoea (which may contain blood and mucus), nausea, fever, abdominal cramps and vomiting. It is spread by the faecaloral route, and the incubation period is usually one to three days.

There are four subgroups of shigella and over 30 serotypes. Serotyping of the organism in NSW is usually performed by Institute of Clinical Pathology and Medical Research (ICPMR) laboratory, Westmead Hospital. Shigellosis is not currently a notifiable condition in NSW. Laboratory staff report that the condition is relatively uncommon in NSW, with fewer than five isolates received by ICPMR for serotyping each month.

Local doctors and laboratories reported an increase in shigellosis diagnoses in March 2000. ICPMR has identified the increase is mainly of Shigella sonnei serotype $\mathrm{G}$ isolates, with over 80 cases identified from early March 2000 until mid-June 2000. This compares to 21 cases in all of 1997, 50 in 1998 and 28 in 1999. Over 90 per cent of cases have been males and most are thought to be gay men between 20-40 years. Some of these are HIV-positive men, several of whom have required treatment in hospital. Further investigations are underway, and a control plan is being implemented.

Clinicians are urged to:

- consider shigellosis in the diagnosis of men with unexplained diarrhoea, and arrange stool examinations where clinically suspected;

- advise persons with diarrhoea to:

- wash their hands thoroughly with soap and running water after using the toilet

- not to handle food for others or have sexual contact with others for at least a week after symptoms have completely resolved;

- antibiotic treatment of laboratory-confirmed shigellosis may help reduce the duration of symptoms and the period of infectiousness. Treatment may therefore be helpful, especially for patients with prolonged illness. St Vincents Hospital in South Eastern Sydney reports that many of the bacteria examined in this outbreak have shown resistance to ampicillin and cotrimoxazole. Where clinically indicated, norfloxacin $400 \mathrm{mg}$ orally, 12-hourly for seven to 10 days is recommended for adult patients associated with this outbreak.

\section{Reference}

1. Therapeutic Guidelines, Antibiotic. 10th edition. Melbourne: Therapeutic Guidelines Limited: March 1998.

\section{CREUTZFELDT-JACOB DISEASE AND OTHER HUMANTRANSMISSIBLE SPONGIFORM ENCEPHALOPATHIES}

NSW Health has adopted the National Health and Medical Research Council (NHMRC) document, Creutzfeldt-Jakob Disease and the Human Transmissible Spongiform Encephalopathies: Guidelines on patient management and infection control, published in December 1995. These Guidelines are to be used by health care facilities to develop infection control and disinfection and sterilisation strategies to reduce the risk of transmission of CreutzfeldtJakob Disease (CJD).

CJD is a rare, transmissible spongiform encephalopathy that is characterised by progressive dementia accompanied by a variety of neurological signs, with an invariably fatal outcome after a clinical course that may last from a few weeks to several years. ${ }^{1}$ CJD occurs sporadically at an annual rate of one person per million per year. ${ }^{3}$

The infectious agents are thought to be prions. Prions are made of an abnormal form of internal cell membrane protein $(\mathrm{PrP})$, which are theorised to be in the transmission of disease..$^{2,3}$ Prions are relatively hardy.

Transmission to humans has been reported to occur after corneal transplants, through contaminated sterotactic instruments and dura mater grafts, and from injection of pituitary-derived growth hormone. Transmission from neurosurgical instruments has been suspected but not conclusively proven. ${ }^{2}$

Potential infectious body fluids and tissue include cerebrospinal fluid, brain, spinal cord, and eye. Other tissues that may be infectious and require cautious handling are lymph glands, kidney, and lung.

\section{References}

1. Brown P. Guidelines for high risk autopsy cases: Special precautions for Creutzfeldt-Jakob Disease. Illinios: College of American Pathologists, 1990.

2. Funk, EA. (1996). Creutzfeldt-Jakob Disease and other Prion (transmittible neurodegenerative) Diseases. APIC Infection Control and Applied Epidemiology: Principles and Practice. St Louis: Mosby, 1996.

3. National Health and Medical Research Council. CreutzfeldtJakob Disease and other Human Transmissible Spongiform Encephalopathies: Guidelines on patient management and infection control. Canberra: Australian Government Publishing Service, 1996. 


\section{FIGURE 6}

\section{REPORTS OF SELECTED COMMUNICABLE DISEASES, NSW, JANUARY 1995 TO JUNE 2000,}

\section{BY MONTH OF ONSET}

These are preliminary data: case counts in recent months may increase because of reporting delays. Laboratory-confirmed cases, except for measles, meningococcal disease and pertussis _ actual _ _ predicted after adjusting for likely reporting delays.

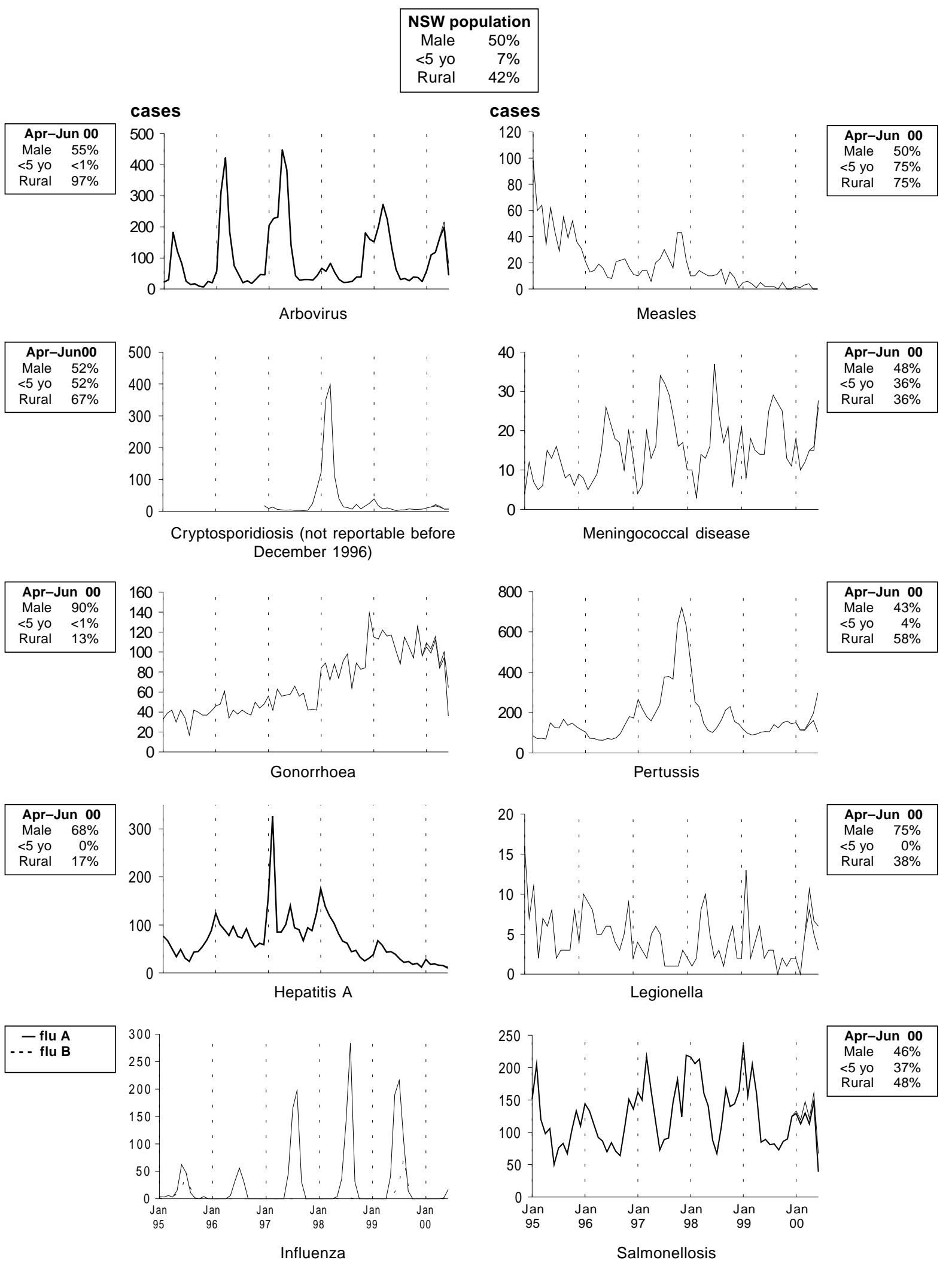

* For definition, see NSW Public Health Bulletin, April 2000 
Condition CSA NSA WSA WEN SWS CCA HUN ILL SES NRA MNC

Blood-borne and sexually transmitted NEA MAC MWA FWA GMA

SA CHS Total

AIDS
HIV infection*

Hepatitis B - acute viral

Hepatitis $B$ - acute virat

Hepatitis C - acute viral

Hepatitis C - other ${ }^{\star}$

Hepatitis D - unspecified

Hepatitis, acute viral (not otherwise specified)

Chancroid* $^{*}$

Chlamydia (genital)*

Gonorrhoe

Syphilis

Arboviral infection (BFV)

Arboviral infection (RRV) $)^{*}$

Arboviral infection (Other)

Zoona

$\begin{array}{cccccccc}1 & 3 & 1 & 1 & - & - & 1 & - \\ - & - & - & - & - & - & - & 2 \\ - & & 1 & 2 & 1 & - & 1\end{array}$

Zoonoses

Brucellosis*
Leptospirosis*

Q fever $^{*}$

Respiratory and othe

Blood lead leve

Legionnaires' Longbeachae*

Legionnaires' Pneumoph

Leprosy

Meningococcal infection (invasive)

Mycobacterial tuberculosis
Mycobacteria other than TB

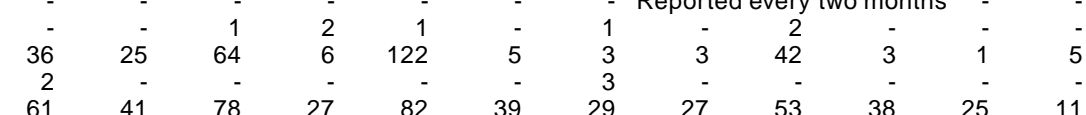

\section{Vaccine-preventable}

Adverse event after immunisation

$H$.influenzae $b$ infection (invasive)

Measles

Mumps*

Pertussis

Rubella*

Faecal-oral

Botulism

Cholera*
Cryptosporidiosis*

Food borne illness (not otherwise specified)

Gastroenteritis (in an institution)

Haemolytic uraemic syndrome

Hepatitis $A^{*}$
Hepatitis $E^{*}$

Listeriosis*

Salmonellosis (not otherwise specified)

Typhoid and paratyphoid*

* lab-confirmed cases only

CSA $=$ Central Sydney Area

$\mathrm{NSA}=$ Northern Sydney Area 